\title{
Intravenous leiomyomatosis with right atrium extension in two patients: A case report
}

\author{
LIANXUE ZHANG ${ }^{1}$, YANG DUAN ${ }^{2}$, FULIN SONG $^{3}$, JUNRUI XIAO $^{2}$ and BENQIANG YANG ${ }^{2}$ \\ ${ }^{1}$ Jinzhou Medical University, General Hospital of Shenyang Military Command Training Base for Graduate; \\ Departments of ${ }^{2}$ Radiology and ${ }^{3}$ Pathology, General Hospital of Shenyang \\ Military Command, Shenyang, Liaoning 110016, P.R. China
}

Received March 25, 2016; Accepted August 8, 2016

DOI: $10.3892 / \operatorname{mco} .2016 .1023$

\begin{abstract}
Intravenous leiomyomatosis (IVL) is a rare benign tumor of the uterus mesoderm, which can spread via the vein, from the micro-vein to the inferior vena cava, and even to the right atrium. IVL was first described this disease in 1896 and the first presented case of IVL with right atrium extension in the autopsy was described in 1907. On rare occasions, the tumors may extend into the regional and systemic veins, thus reaching the heart. This may subsequently cause intracardiac leiomyomatosis (ICL), which may lead to congestive heart failure and on occasion, sudden fatalities. Due to its rarity and diffuse symptoms, the misdiagnosis of ICL is common and as a result, the condition may be under-reported. The present study reported two cases of IVL resected at the General Hospital of Shenyang Military Command. A hysteromyomectomy for uterine fibroids was performed on each patient (Case 1, 41 years ago; Case 2, 3 years ago). One patient presented with chest pain following a period of activity and the other presented with heart neoplasm during a routine health examination.
\end{abstract}

\section{Introduction}

Intravenous leiomyomatosis (IVL) is a rare tumor, which is usually of uterine origin. Birch-Hirschfeld (1) first described this disease in 1896, and following its discovery, Dürck (2) reported IVL with right atrium extension in the autopsy. This tumor type is characterized by intravascular nodular masses of histologically benign smooth muscle that may extend variable distances, including into the inferior vena cava, right atrium and pulmonary arteries. The tumor may arise from uterine leiomyoma, walls of uterine vessels, or myometrium.

Correspondence to: Professor Benqiang Yang, Department of Radiology, General Hospital of Shenyang Military Command, 83 Wenhua Road, Shenyang, Liaoning 110016, P.R. China E-mail: bqyang888@sina.com

Key words: intravenous leiomyomatosis, right atrium extension, computed tomography
The age of presentation is usually between 20 and 70 years, with a mediun age of 45 years. The most commonly affected women are pre-menopausal and multiparous. Intracardiac extension may represent a diagnostic challenge, as it is usually misdiagnosed as a right atrial myxoma and may cause multiple symptoms, including shortness of breath, tachycardia, chest pain, syncope and even mortality. The present study reported two separate cases of IVL resected at the General Hospital of Shenyang Military Command.

\section{Case report}

Case 1. A 60-year-old female was admitted to General Hospital of Shenyang Military Command due to a right atrium neoplasm identified during examination. A hysteromyomectomy for uterine fibroids was performed 41 years ago. Following the operation, the patient stopped menstruating. The patient was pregnant twice, however, only gave birth to one child. An echocardiogram revealed a neoplasm in the inferior vena cava and right atrium. Computed tomography (CT) revealed a neoplasm in the right pelvic cavity, which had invaded into vena iliaca interna, common iliac vein and inferior vena cava, finally reaching into right atrium. The neoplasm was round-like in the right atrium (Fig. 1A). The tumor spread via the vein system and was shown low density in the vein system. Following surgery, gross pathology of two sections of smooth grey white mass demonstrated sections of calcification. These masses measured $9.0 \times 4.5 \times 3.0 \mathrm{~cm}$ (Fig. 1B). Following surgery, microscopy revealed a muscle-like cell arranged in a cord-like formation and shaped as a long fusiform and nucleus shaped long rod with an abundant blood supply (Fig. 1C). The diagnosis was intravenous leiomyomatosis (IVL) with right atrium and inferior vena cava extension. The patient recovered 39 days after surgery. Regrettably, no follow up was performed.

Case 2. A 49-year-old female presented with frequent chest pain after activity over the last half-month. A hysteromyomectomy for uterine fibroids was performed two years ago. The patient stopped menstruating one year ago. She was pregnant once and gave birth to the child. An echocardiogram revealed a neoplasm in the right atrium. CT revealed a right atrium neoplasm with a smooth surface, stretched inferior vena 

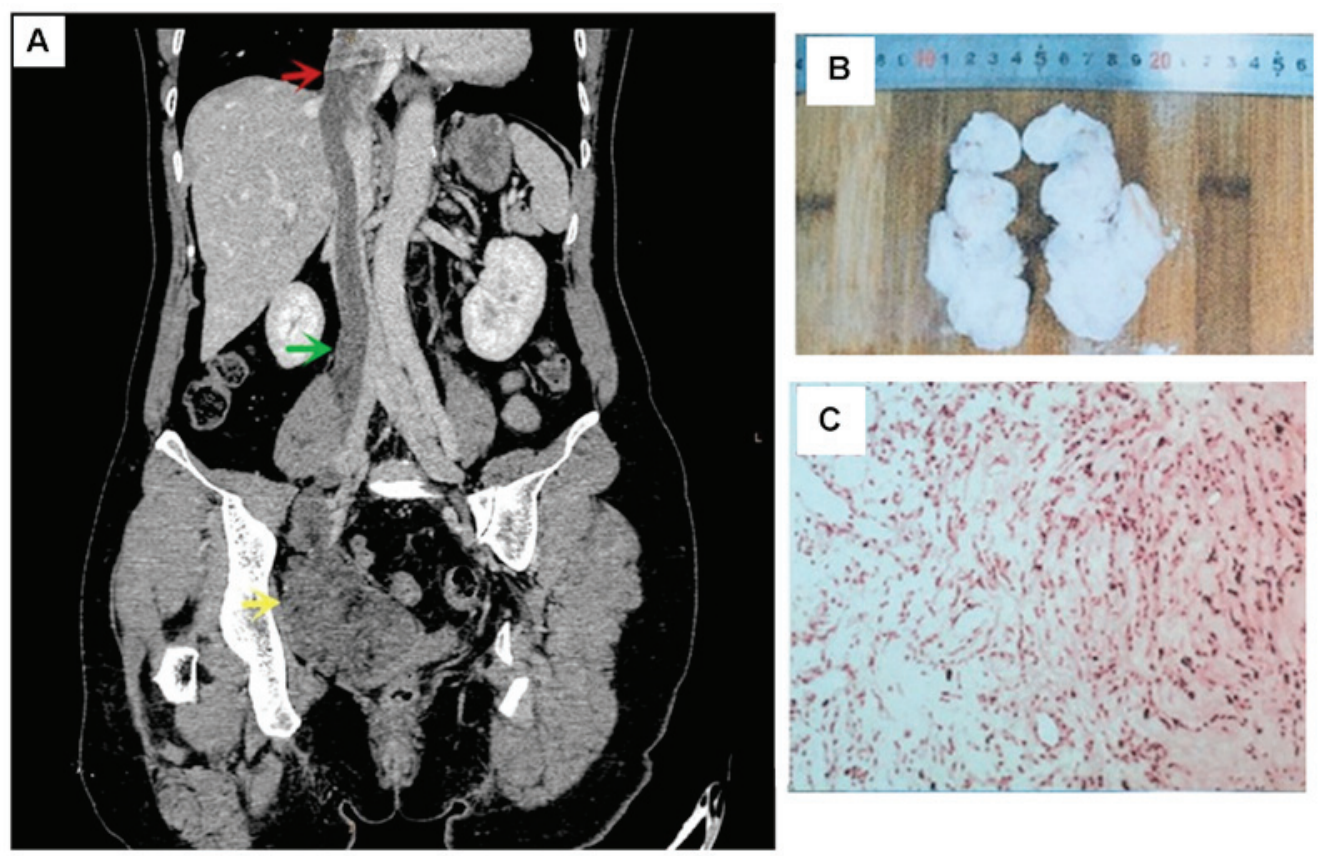

Figure 1. Examination images for case 1. (A) Coronal reformat image of a contrast-enhanced abdomen and pelvis CT scan. An enlarged myomatous uterus with multiple large heterogeneous enhancing masses (yellow arrow) was observed. Tumor extension into the right vena iliaca interna (green arrow) and to the partially visualized right atrium (red arrow) was also observed. (B) Gross pathology of two pieces of smooth grey white mass demonstrated sections of calcification. These masses measured $9.0 \times 4.5 \times 3.0 \mathrm{~cm}$. (C) Histological images revealed cigar-shaped elongated nuclei and muscle fibers without mitosis or atypia, suggestive of smooth muscle cell (hematoxylin and eosin; magnification, x200). CT, computed tomography.
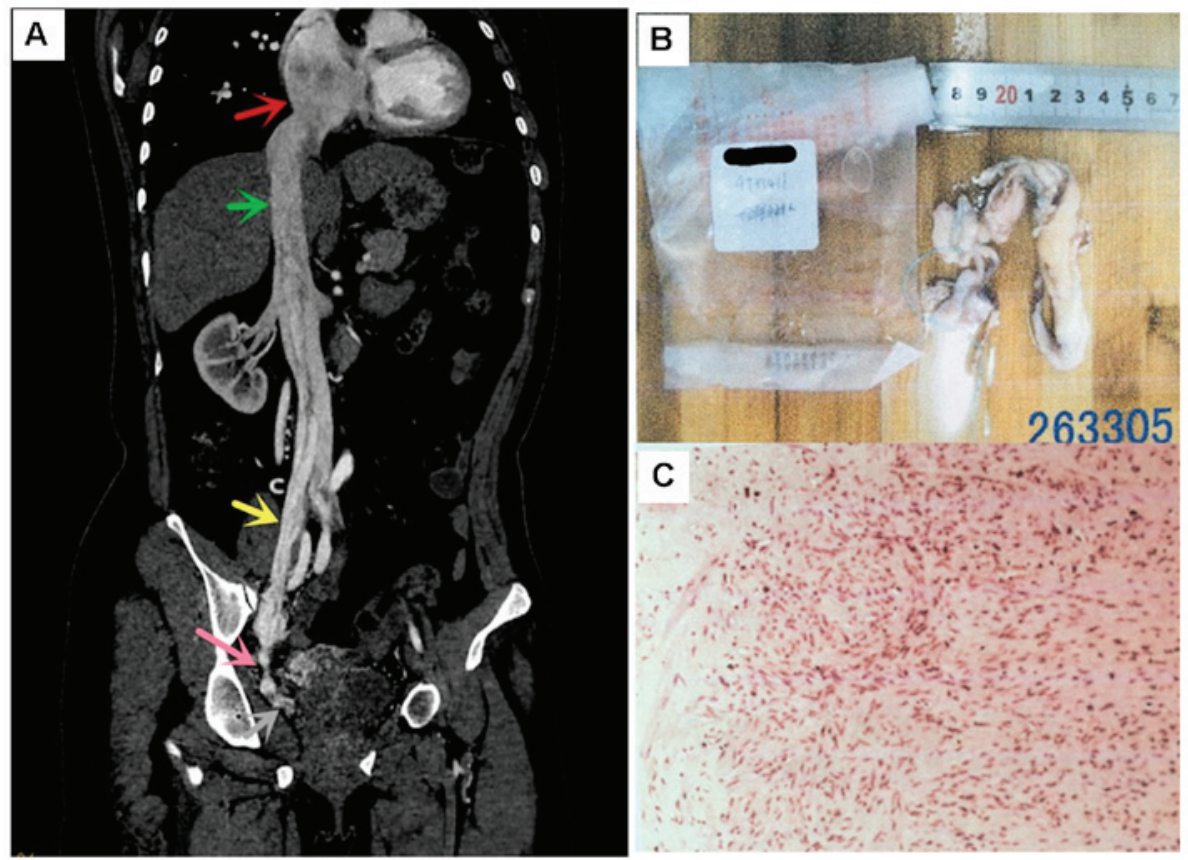

Figure 2. Examination images for case 2. (A) Coronal reformat image of a contrast-enhanced abdomen and pelvis CT scan. Tumor extension into the inferior vena cava (pink arrow), right vena iliaca externa (yellow arrow), vena iliaca interna (green arrow) and to the partially visualized right atrium (red arrow) was observed. (B) Gross pathology using pieces of smooth grey white and grey red mass were observed. One mass measured $9.0 \times 4.5 \times 3.0 \mathrm{~cm}$ and the others measured $4.0 \times 3.5 \times 1.5 \mathrm{~cm}$ in total. (C) A histological image revealed cigar-shaped elongated nuclei and muscle fibers without mitosis or atypia, suggestive of smooth muscle cell (hematoxylin and eosin; magnification, x200) CT, computed tomography.

cava, vena iliaca interna and vena iliaca externa (Fig. 2A). Following surgery, gross pathology was shown in sections of smooth grey white and grey red masses. One mass measured $9.0 \times 4.5 \times 3.0 \mathrm{~cm}$ and the others measured $4.0 \times 3.5 \times 1.5 \mathrm{~cm}$ in total (Fig. 2B). Histology revealed muscle-like cell arranged in a cord-like formation and shaped as a long fusiform and nucleus shaped long rod (Fig. 2C). The diagnosis was IVL with right atrium and inferior vena cava extension. The patient recovered 28 days later. Regrettably, no follow up was performed. 


\section{Discussion}

The symptoms of IVL are atypical and have no specialty; therefore, it is often clinically misdiagnosed. Clinical features included pelvic mass, irregular uterus enlargement and bleeding in the vagina, menstruation variation, abdominal distension, and pelvic cavity compression. Before the tumor spreading via the inferior vena cava, all clinical symptoms were hidden and led to misdiagnosis by physicians. However, when the tumor extended to the right atrium, this caused palpitation, chest pain, discontinuous syncope, sudden mortality and even pulmonary transfer (3).

IVL is a rare disease and the CT features of IVL were unique, particularly in cases of mass with heart extension. At an early stage, contrast-enhanced CT and CT vein (CTV) imaging can distinguish IVL. In non-contrast-enhanced CT images, a mass consisting of an isodense lesion was revealed in the vein and heart lumen; and an off-centered filling-defect was revealed in the vein and heart lumen. The mass was dissociated in the lumen with low density and medium heterogeneous enhancement in the contrast-enhanced CT image. The involved the thickening and extension of the vein system in the lumen $(4,5)$. CTV imaging revealed the mass, which performed vascular reconstruction more accurately and directly. The CTV image confirmed the correlation between the tumor and other viscera vessels, collateral vessels, which was significant for pre-operation evaluation and operation strategy.

For IVL with right atrium extension, differential diagnosis includes vascular thrombus, as well as primary and metastatic tumor types. Vascular thrombus was always without enhancement in the contrasted CT scanning. Metastatic differential diagnosis includes metastasis with IVC invasion (e.g. renal cell carcinoma, adrenal cortical carcinoma, hepatocellular carcinoma and lymphoma), atrial myxoma, right-sided heart thrombus or embolus, and leiomyosarcoma. Atrial myxoma is usually the initial diagnosis in these patients given that it is the most common primary heart tumor. However, 60-75\% of atrial myxomas are located in the left atrium attached to atrial septum, usually at the fossa ovalis, and no IVC extension exists unless it arises from the IVC proper (in extremely rare cases) $(6,7)$. Another diagnosis to consider is benign metastasizing leiomyomas (BML). This disease is characterized by uterine leiomyoma in young adulthood, with pulmonary metastasis occurring in the pre-menopausal period (8).

Treatment of IVL is almost always surgical (9-11), which includes excision of the extra-uterine tumor and myomectomy, or total hysterectomy, as necessary. The tumor usually spreads via the veins. Therefore, once intravenous leiomyomatosis was suspected, the vein system must be assessed completely and the tumor was excised as completely as possible. As for patients where resection is not possible or where the tumor remains, anti-estrogen treatment was a good choice. Anti-estrogen existed in uterine vascular leiomyoma. The level of estrogen in the uterine vascular leiomyoma was 10-times higher compared with healthy tissue. Kokawa et al (12) suggested that higher estrogen and estrogen receptor levels may influence the growth and invasion of the tumor.

In the present study, IVL, a rare diagnosis that merits consideration in young pre-menopausal or old post-menopausal female patients with cardiac symptoms associated with a right atrial mass. From the two-presented cases, it was clear that radiologists serve a vital role in the diagnosis and follow-up of patients with the diagnosis of IVL. Once patients present with a uterine or periuterine mass, and deep vein thrombus at the same time, IVL must be considered. Early detection and correct diagnosis is imperative for appropriate treatment and surgical planning.

\section{References}

1. Birch-Hirschfeld FV (ed): Lehrbuch der Pathologischen Anatomie. $5^{\text {th }}$ edition. F.C.W. Vogel, Leipzig, p226, 1896 (In German).

2. Durck H: Ueber ien kontinvierlich durch die entere holhlvene in das herz vorwachsendes: Fibromyom des uterus. Munch Med Wochenschr 54: 1154, 1907. (In German).

3. Lam PM, Lo KW, Yu MY, Wong WS, Lau JY, Arifi AA and Cheung TH: Intravenous leiomyomatosis: Two cases with different routes of tumor extension. J Vasc Surg 39: 465-469, 2004.

4. Oliveira L and Ramos S: Anesthetic approach for a clinical case of intravenous leiomyomatosis: Case report. Braz J Anesthesiol 63: 504-507, 2013.

5. Demirkiran F, Sal V, Kaya U, Alhan C and Tokgozoqlu N: Intravenous leiomyoma with extension to the heart: A case report and review of the literature. Case Rep Obstet Gynecol 2013: $602407,2013$.

6. Grebenc ML, Rosado-de-Christenson ML, Green CE, Burke AP and Galvin JR: Cardiac myxoma: Imaging features in 83 patients. Radiographics 22: 673-689, 2002.

7. Buckley O, Madan R, Kwong R, Rybicki FJ and Hunsaker A: Cardiac masses, part 2: Key imaging features for diagnosis and surgical planning. AJR Am J Roentgenol 197: W842-W851, 2011.

8. Awonuga AO, Rotas M, Imudia AN, Choi C and Khulpateea N: Recurrent benign metastasizing leiomyoma after hysterectomy and bilateral salpingo-oophorectomy. Arch Gynecol Obstet 278: 373-376, 2008.

9. Sogabe M, Kawahito K, Aizawa K, Sato H and Misawa Y: Uterine intravenous leiomyomatosis with right ventricular extension. Ann Thorac Cardiovasc Surg 20 (Suppl): S933-S936, 2014.

10. Kang LQ, Zhang B, Liu BG and Liu FH: Diagnosis of intravenous leiomyomatosis extending to heart with emphasis on magnetic resonance imaging. Chin Med J (Engl) 125: 33-37, 2012.

11. Clay TD, Dimitriou J, McNally OM, Russell PA, Newcomb AE and Wilson AM: Intravenous leiomyomatosis with intracardiac extension-a review of diagnosis and management with an illustrative case. Surg Oncol 22: e44-e52, 2013.

12. Kokawa K, Yamoto M, Yata C, Mabuchi Y and Umesaki N: Postmenopausal intravenous leiomyomatosis with high levels of estradiol and estrogen receptor. Obstet Gynecol 100: 1124-1126, 2002. 